\title{
Spin Dependent Electron Tunneling in a Ferromagnet Superconductor Ferromagnet Junction
}

\author{
L. Kowalewski and R.J. WojCIECHOWSKI \\ ${ }^{a}$ Faculty of Physics, Adam Mickiewicz University \\ Umultowska 85, 61-614 Poznań, Poland
}

\begin{abstract}
The coherent spin polarized transport in ferromagnet/superconductor/ferromagnet double barrier junctions is analyzed. Using the Bogolubov-de Gennes equation with appropriate boundary conditions, we calculate probabilities of spin dependent transport processes in the ferromagnet/superconductor/ferromagnet junction. In particular, we discuss the nonlocal processes such as the crossed Andreev reflection and elastic co-tunneling. These processes contribute to tunneling a current when the distance between the two magnetic electrodes is comparable to the superconducting coherence length. The dependences of the tunneling transport processes on the strength of the exchange field in the ferromagnetic electrodes, and on the height of the tunnel barriers are presented.
\end{abstract}

PACS numbers: 74.45.+c, 74.50.+r, 73.23.Ad

\section{Introduction}

In the last few years spin dependent transport has been of particular interest both for fundamental physics as well as for promising spintronics applications. In this paper, we concentrate on spin dependent charge transport through the ferromagnet/superconductor/ferromagnet $(\mathrm{F} / \mathrm{S} / \mathrm{F})$ junction. The charge transport is decomposed into two processes. The first type, which can be named local processes, consists of the normal and Andreev reflections from the first $\mathrm{F} / \mathrm{S}$ interface. These reflections occur in the single normal- or ferromagnetic metalsuperconductor junction. The second one, nonlocal processes, occurs in a region where there is no drive current. It is characteristic of multi-terminal junctions [1] and consists of the crossed Andreev (CAR) reflection and elastic co-tunneling (EC) [2-8]. It has been shown $[1,8]$ that when the distance $l$ between the ferromagnetic electrode metals is smaller than or comparable to the superconducting coherence length $\xi$, an injection of an electron (or hole) with a subgap energy leads to the additional nonlocal transport processes named the CAR and EC [2]. The experimental evidence for both CAR and EC has been observed by Russo et al. [3] and by Beckmann and Löhneysen [4]. These processes are phase coherent and appear in $\mathrm{F} / \mathrm{S} / \mathrm{F}$ double tunnel junctions when the thickness of the superconducting $(\mathrm{S})$ interlayer is small enough. If the thickness of S layer is large enough, a double tunnel junction can be regarded as two independent single $\mathrm{F} / \mathrm{S}$ junctions and then the tunneling processes are incoherent [6]. In addition, the total electronic current in the $\mathrm{F} / \mathrm{S} / \mathrm{F}$ junction strongly depends on the relative orientation of the magnetization of two ferromagnetic electrodes $[7,8]$. The CAR is a consequence of capturing an- other electron (or hole) from the right electrode in order to form a Cooper pair in the superconductor. A simultaneously appropriate hole (or electron) is reflected in the right electrode. The EC consists on the direct tunneling of the injected particle from one electrode to the second one due to the proximity effect from the superconductor without the formation of the Cooper pair [5]. The electron-like quasiparticle penetrates a depth $\approx \xi$ into the superconductor before its conversion into the condensate of the Cooper pair. The charge current $2 e v_{\mathrm{F}}$ in the ferromagnetic metals disappears, as an appropriate quasiparticle current $J_{Q}$ in the $\mathrm{S}$ layer, according with the formula $J_{Q}=2 e v_{\mathrm{F}} \exp (-y / \xi)$, where the coherence length is given by $\xi=\hbar v_{\mathrm{F}} /\left(2 \sqrt{\Delta^{2}-E^{2}}\right.$. Simultaneously, $J_{Q}$ converts into an increasing supercurrent of the Cooper pairs $J_{\mathrm{s}}=2 e v_{\mathrm{F}}[1-\exp (-y / \xi)]$. In this paper, we discuss how the nonlocal effects are influenced by the energy of the incident electron and the width of the superconducting layer. These effects are important for the following reasons:

1. The spin polarized current in the metallic $\mathrm{F} / \mathrm{S} / \mathrm{F}$ heterostructures, due to the CAR, can be applied in spintronics by implementation of magnetoresistive devices, such as spin switches or memory elements.

2. The spin polarized subgap tunneling spectroscopy in double $\mathrm{F} / \mathrm{S} / \mathrm{F}$ junctions provides information on the pairing symmetry in ferromagnetic superconductors.

3. The CAR enables the realization of solid state entanglers by the creation of a mobile entangled pairs of electrons in spatially separated nanoelectronic circuits. 


\section{Model and calculations}

We consider an F/S/F double junction, consisting of two ferromagnetic metallic electrodes and a superconducting layer separated from the electrodes by thin insulating interfaces. Here, to avoid unnecessary complication, we consider a model of $\mathrm{F} / \mathrm{S} / \mathrm{F}$ junction with a clean superconductor. In the clean limit we can easily solve the Bogolubov-de Gennes (BdG) equations within the approach proposed in [9]. The wave function, describing the quasiparticle propagation across the $\mathrm{F} / \mathrm{S} / \mathrm{F}$ junction, can be obtained from the extended $\mathrm{BdG}$ equations. In the ferromagnetic regions, these equations are reduced to appropriate Schrödinger equations. The extended BdG equations take the following form:

$$
\begin{aligned}
& \left(\begin{array}{cc}
H_{0}-E-\sigma h_{\mathrm{ex}}, & \Delta \\
\Delta^{\dagger} & -\left(H_{0}-E+\sigma h_{\mathrm{ex}}\right)
\end{array}\right)\left(\begin{array}{c}
f_{\sigma}(\boldsymbol{r}) \\
g_{-\sigma}(\boldsymbol{r})
\end{array}\right) \\
& =\check{O},
\end{aligned}
$$

where $H_{0}=-\hbar^{2} / 2 m \nabla^{2}-E_{\mathrm{F}}+W(\boldsymbol{r})$ is the single-particle Hamiltonian, $E$ is the quasiparticle energy measured from the Fermi energy $E_{\mathrm{F}}$, and $\sigma=+(-1)$ denotes the up (down) spin subband. $W(\boldsymbol{r})$ stands for the interface potential and is measured by the dimensionless parameter $z=2 m W / \hbar^{2} k_{\mathrm{F}}$. For the ferromagnetic electrodes, we adopt the Stoner model. Thus, the exchange field in both the left $(y<-l / 2)$ and the right electrodes $(y>l / 2)$ can be defined as follows:

$$
h_{\mathrm{ex}}=\left\{\begin{array}{cc}
h_{0}, & y<-l / 2, \\
0, & -l / 2<y<+l / 2, \\
\pm h_{0}, & y>l / 2,
\end{array}\right.
$$

where $+h_{0}$ and $-h_{0}$ define the exchange fields for the parallel and antiparallel configuration, respectively. The solution of Eq. (1) in the two electrodes corresponding to the injection of an electron with spin $\sigma$ (from one, e.g. the left ferromagnetic electrode) with energy $E>0$, spin $\sigma$ and angle of incidence $\Theta$ can be written in the following form:

$$
\begin{aligned}
& \Psi_{\sigma}^{(y<-l / 2)}(y)=\left(\begin{array}{c}
1 \\
0
\end{array}\right) \mathrm{e}^{\mathrm{i} k_{\sigma}^{+} y}+a_{-\sigma}\left(\begin{array}{l}
0 \\
1
\end{array}\right) \mathrm{e}^{\mathrm{i} k_{-\sigma}^{-} y} \\
& +b_{\sigma}\left(\begin{array}{l}
1 \\
0
\end{array}\right) \mathrm{e}^{-\mathrm{i} k_{\sigma}^{+} y} \\
& \Psi_{\sigma}^{(y>l / 2)}(y)=c_{\sigma}\left(\begin{array}{l}
1 \\
0
\end{array}\right) \mathrm{e}^{\mathrm{i} k_{\sigma(-\sigma)}^{+} y} \\
& +d_{\sigma}\left(\begin{array}{c}
0 \\
1
\end{array}\right) \mathrm{e}^{-\mathrm{i} k_{-\sigma(\sigma)}^{-} y}
\end{aligned}
$$

where the $y$ component of the momentum for an electron $(+)$ and hole $(-)$ takes the form: $k_{\sigma}^{ \pm}=$ $\sqrt{\left(2 m / \hbar^{2}\right)\left(E_{\mathrm{F}}+\sigma h_{0} \pm E\right)-\boldsymbol{k}_{\|, \sigma}^{2}}$, and the momentum parallel to the interface has the form: $\left|\boldsymbol{k}_{\|, \sigma}\right|=$ $\sqrt{\left(2 m / \hbar^{2}\right)\left(E_{\mathrm{F}}+\sigma h_{0}+E\right)} \sin (\Theta)$. The probability amplitudes of the transport processes $a_{\sigma}(E, \Theta), b_{\sigma}(E, \Theta)$, $c_{\sigma}(E, \Theta), d_{\sigma}(E, \Theta)$ determine the ordinary Andreev and normal reflections, elastic co-tunneling, and crossed Andreev reflection, respectively [5-8]. The interference of quasiparticles in the superconducting layer leads to oscillations of all the transport processes if the following resonance condition is fulfilled $q_{\sigma}^{+}-q_{\sigma}^{-}=2 n \pi / l$, where the momentum $q^{ \pm}$in the $\mathrm{S}$ is given by $q_{\sigma}^{ \pm}=$ $\sqrt{\left(2 m / \hbar^{2}\right)\left(E_{\mathrm{F}} \pm \sqrt{E^{2}-\Delta^{2}}\right)-\boldsymbol{k}_{\|, \sigma}^{2}}$. The coefficients $a_{\sigma}(E, \Theta), b_{\sigma}(E, \Theta), c_{\sigma}(E, \Theta), d_{\sigma}(E, \Theta)$ are determined by the appropriate boundary conditions for the wave functions and their derivatives at both the interfaces placed at $y= \pm l / 2$. Although it is true that in experiments one does not measure directly the coefficients $a, b, c, d$, however, these coefficients determine the charge transport through $\mathrm{F} / \mathrm{S} / \mathrm{F}$ junctions and the pairing symmetry of the superconductor. For example, the sign (plus or minus) of the voltage measured across the second $\mathrm{S} / \mathrm{F}$ tunnel barrier of the junction $\mathrm{F} / \mathrm{S} / \mathrm{F}$ depends on whether EC or CAR occurs with larger probability [3]. Similarly, in a case of half-metallic ferromagnet electrodes the process of CAR can be completely suppressed while EC will be allowed or vice versa, depending on the mutual alignments (parallel or antiparallel) of the electrode magnetizations. Further calculations of the charge and spin currents are in progress.

\section{Results and conclusions}

The coherent charge transport through the $\mathrm{F} / \mathrm{S} / \mathrm{F}$ junction exhibits many characteristic features both for the subgap transmission and for energies greater than superconducting gap. The important role, in the transport processes through the double junction, plays both the pairing symmetry of a superconducting state and the magnetic orientation of the ferromagnetic electrodes [10]. For example, in the case of the spin singlet pairing and the parallel configuration of the electrodes, the normal and crossed Andreev reflections are suppressed and for ferromagnetic half-metals $\left(x=h_{0} / E_{\mathrm{F}}=1\right)$ these processes are completely blocked. In the case of antiparallel alignment, the crossed Andreev reflection is favored while the normal Andreev reflection is suppressed (or blocked for the ferromagnetic half-metal). In the paper, we have calculated the probabilities of all the components of the coherent transport processes, namely: the ordinary Andreev, crossed Andreev and normal reflections $\left(|a|^{2},|d|^{2}\right.$, $\left.|b|^{2}\right)$, as well as the direct tunneling through the double junction $\left(|c|^{2}\right)$. The sum of these probabilities is normalized to 1 . The numerical calculations were performed for wide ranges of the model parameters, i.e. for the strengths of the exchange field $x$, the heights of the interfacial barriers $z$, and the thicknesses of the superconducting layer $l$. As we can see in Figs. 1-3, if the energy of the incident electron is greater than the superconducting gap $\Delta$, all these probabilities oscillate as the functions of $E / \Delta$ and $l$ due to the interference of incoming and outgoing particles. If the thickness of the superconductor is small in comparison with the superconducting coherence length (see Fig. 1) the nonlocal processes (EC and 

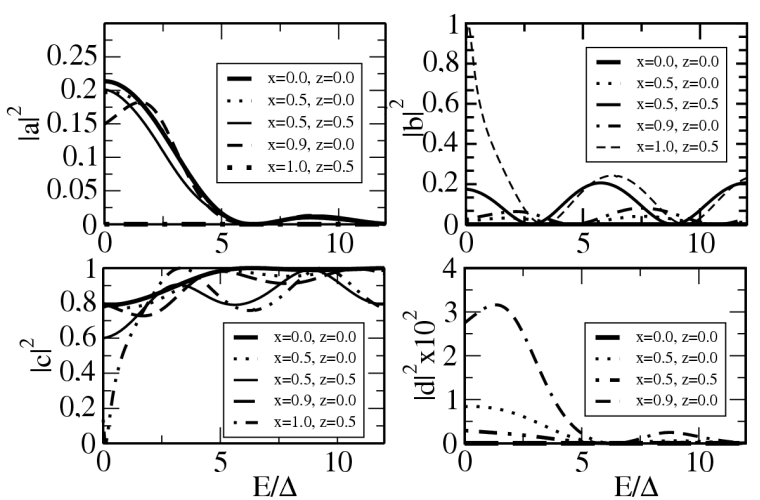

Fig. 1. The probability of the four transport processes as a function of $E / \Delta$ for $l k_{\mathrm{F}}=10^{3}$ and for several values of $x$ and $z$.
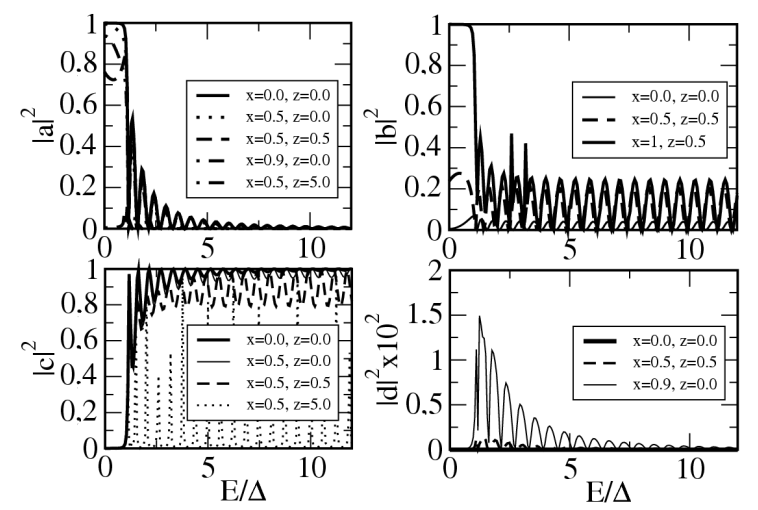

Fig. 2. The probability of the four transport processes as a function of $E / \Delta$ for $l k_{\mathrm{F}}=10^{4}$ and for several values of $x$ and $z$.

CAR) play important role for subgap energies. In the opposite limit, i.e. if the thickness of the superconductor is large (see Fig. 2), suppression of EC and CAR is observed. Changes of the tunneling current in double junctions for different widths of the superconducting layer were observed in [3]. The maxima (minima) of the elastic co-tunneling correspond one to one to minima (maxima) of the ordinary Andreev, crossed Andreev and normal reflections. If the thickness $l \geq \xi$, then the non-local processes decay. When the EC dominates i.e. when the charge carriers pass from the left to the right electrode without conversion into the supercurrent, the normal and Andreev and crossed Andreev reflections decay at the both interfaces. It is worthy mentioning that typical ranges of the coherence length are of the order of $\xi=$ $10 \AA$ and $\xi=1.0 \mu \mathrm{m}$ for high- $T_{\mathrm{c}}$ and low-temperatures superconductors, respectively. The electron-beam lithography technique allows one to fabricate devices which have sizes of the order of tens $\mathrm{nm}$.

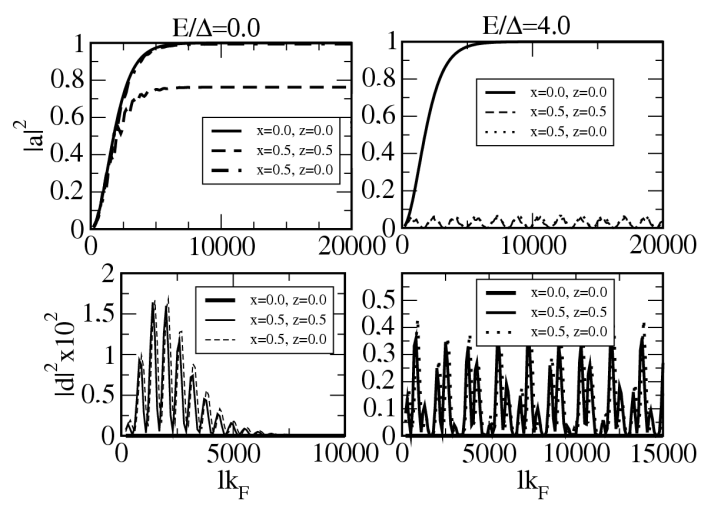

Fig. 3. The probability of the ordinary $\left(|a|^{2}\right)$ and crossed Andreev $\left(|d|^{2}\right)$ reflections as a function of $l k_{\mathrm{F}}$ for $E / \Delta=0.0$ (left part) and $E / \Delta=4.0$ (right part) and for several values of $x$ and $z$.

\section{References}

[1] J.P. Morten, A. Brataas, W. Belzig, Phys. Rev. B 74, 214510 (2006).

[2] J.M. Byers, M. Flatté, Phys. Rev. Lett. 74, 306 (1995).

[3] S. Russo, M. Kroug, T.M. Klapwijk, A.F. Morpurgo, Phys. Rev. Lett. 95, 027002 (2005).

[4] D. Beckmann, H. v. Löhneysen, AIP Conf. Proc. 850, 875 (2006).

[5] J.M. Božević, Z. Radović, Phys. Rev. B 66, 134524 (2002).

[6] Z.C. Dong, R. Shen, Z.M. Zheng, D.Y. Xing, Z.D. Wang, Phys. Rev. B 67, 134515 (2003).

[7] T. Yamashita, H. Imamura, S. Takahashi, S. Maekawa, Phys. Rev. B 67, 094515 (2003).

[8] T. Yamashita, S. Takahashi, S. Maekawa, Phys. Rev. B 68, 174504 (2003).

[9] G.E. Blonder, M. Tinkham, T.M. Klapwijk, Phys. Rev. B 25, 4515 (1982).

[10] C. Benjamin, Phys. Rev. B 74, 1805503 (2006). 